\title{
Needle Stick And Sharp Related Injuries- Ethical Considerations, Prevention, And Management
}

Faisal Hanif, Umar Khurshid, Haroon Sabir Khan, Muhammad Zill-e-Humayun Mirza

Laboratory and Health care workers (HCW) are exposed to many occupational related hazards. Both are at considerable risk of acquiring infections. Needle stick/prick injuries (NSIs) can lead to blood borne infections such as HIV, Hepatitis B and Hepatitis C. NSIs are one of the most common biological hazards inside laboratory. These injuries can be sustained while handling a sharp or needle and more commonly during recapping and waste disposal. Risk of transmission after these injuries from infected patients to $\mathrm{HCW}$ are $3-30 \%$ for hepatitis $\mathrm{B}, 3 \%$ for hepatitis $\mathrm{C}$, and $0.3 \%$ for $\mathrm{HIV}^{1}$.

It is pertinent to mention that frequency of needle stick injuries is highest among nursing staff as reported by many published studies ${ }^{2}$. Most common causes of injury among indoor hospital patients is phlebotomy or intravenous infusions. Still the exact magnitude of NSI in Pakistan is not clear because of poor or almost no reporting, surveillance system exist ${ }^{2,3}$.

Needle stick injuries (NSI) are responsible mainly for the transmission of blood related diseases. Hospital staff including doctors, nurses, and paramedics are at highest risk of such injuries. It is said that almost one half of the intravenous infusions in our region are hazardous not only for patients but also for health care provider. Recapping of syringe after use has been considered as the most frequent factor responsible for NSI although there are different causes of needle stick injuries. Accidental pricks in such cases are more likely to be by used infected needle; therefore, they are at considerable risk of acquiring such infections. In Pakistan, hepatitis B and $\mathrm{C}$ are the two main infectious diseases that can be transmitted through prick of infected needles and probability of hepatitis B transmission is 3$30 \%{ }^{4}$.

It is estimated that performing phlebotomy to draw blood for laboratory investigations is the most common cause of needle stick injury in hospital wards ${ }^{5}$.

\section{Ethical considerations in Needle stick injuries:}

Apart from the academic and case management point of

I Faisal Hanif, ------------7

I PNS SHIFA Karachi

I Umar Khurshid,

I AFIP Rawalpindi.

I Haroon Sabir Khan,

I PNS SHIFA Karachi

I Muhammad Zill-e-Humayun Mirza

I PNS SHIFA Karachi

I Received: 13-08-2018

I Accepted: 26-09-2018 view; one pertinent aspect remains the non-presence of an explicit occupational exposure law dealing with such issues. The situation is complicated even further when it is analyzed that any post-exposure prophylaxis (PEP) rests on the presence or absence of the infecting agent in the source patient. Consider a situation in which the source patient or the attendants are unwilling to get the test done. Would it be ethical enough to perform an unconsented test on the individual's previously held samples? This, at times, may create an ethical issue - a right of the source patient to choose or decline a medical advice and the right of the health care worker in perusing a logical and recommended course of PEP after the source patient's status is ascertained. Realistically both the involved parties have stakes, risks and fears that need to be timely and elaborately addressed and discussed.

Most patients are happy to give permission for their blood to be tested but few refuses. It is because of the fears about its implications on jobs, health insurance and least but not the last they are afraid of social stigma and discrimination 6 .

The complexity is not only of getting an informed consent; it's also because of the fact that asymptomatic carriage of infections is common. A quarter of HIV infected patients don't know about their disease and $26 \%$ of people cannot identify any particular risk factor. Any positive test on an unconsented sample is going to be followed by certain reactions. Revealing such a positive status to an individual may potentially make the patient liable to severe psychological stress apart from the social and physical effects this disclosure is going to have on them. The patient may perceive it as a violation of privacy and autonomy; fears of social stigma, tainted repute and social discrimination are well found concerns of the patient ${ }^{7}$. The resulting anxiety, of an unprepared mind, may even lead to distrust between the caregivers and the patient - a medical care avoiding behavior- none serving the interests of the patient or the people around the patient, initiate protective behavior in the source patient in which epidemiologically relevant information may be concealed and distorted - none helping the public health staff.

Whatever course of discussion is adapted and whatever course of ethical theories, be it the deontological approach or the utilitarian approach, be debated, the health care provider has to weigh the harms and benefits to both the source patient and the health care worker who by now has become a patient too.

Even in the West the opinion remains divided on the legality 
Needle Stick and Sharp related injuries- Ethical considerations, prevention, and management

of unconsented testing in such circumstances. Law in the US is divided with some states mandating unconsented testing while others restricting such use ${ }^{8}$. General Medical Council in the UK also favours the second option. For us the quagmire is complex, with no relevant laws on the subject issue. However following are suggested to tackle the situation at hand.

1. All efforts can be made to explain the purpose, risks and benefits of the particular test. This can be preferably done by the health care provider supposed to treat the exposed patient. In situations where the patient is unwilling for consent the applicability of unconsented testing of any retained sample be explained to the patient.

2. If after all the deliberation the patient remains unconvinced - permission be granted for the unconsented sample testing. The authority conceding such test has to be identified and procedure for the documentation of such an event be devised. Feedback of the tested sample to the patient remains the right of the source patient who will be asked how to get informed of the results. Proper protocols to be made in facilities which embark upon the testing of unconsented testing.

3. When unconsented specimen is tested patient confidentiality has to be respected.

4. Is it ethical to allow Health care worker (doctors, nurses and paramedics) to work with sharps/needles without proper training to prevent sharp related injuries. Training on Universal Precaution Guidelines, protocols regarding post-exposure prophylaxis, and safety devices has to be provided to prevent such injuries among Health care workers.

\section{Needle Stick Injury Protocol}

In the following section we will discuss about the protocol to be followed after needle stick injury. ${ }^{1,9,10}$

\section{Immediate care (On spot)}

1. First step is to wash injured part with tap water and soap

2. If there is any exposure to mucosa like eyes, or if the wound size is large, irrigation with plentiful amounts of normal saline.

3. Use of bleach or antiseptics to clean area will not help.

\section{Hospital Care at emergency department}

1. First step is to irrigate and clean the wound.

2. Risk assessment of the patient

3. Next step is to assess if tetanus and/or hepatitis B prophylaxis is required or not. Health care staff are mostly vaccinated against hepatitis $\mathrm{B}$.

4. It is the responsibility of employers to ensure that all vaccines and post exposure prophylaxis remain available to the employee at all time and at a reasonable location and at no cost.

\section{Prophylaxis for HIV with three-step risk assessment approach}

Assess necessity for anti-retrovirals based on an assessment of the risk by using the three-step approach as developed by the Center for Disease Control and Prevention (CDC).

\section{Step 1: Determine exposure code}

1. Do not panic and immediately expose and express the wound and encourage oozing of blood. Next inquire about the source material, it might be blood, body fluid or any potentially infectious material. If none of above, there is no risk of HIV transmission. If yes than what type of exposure has occurred

2. If the skin is intact and there is no visible injury, there is no risk of HIV transmission.

3. If the mucous membrane was exposed and the volume of fluid few drops, several drops or major splash? If few drops, the category is exposure code one. If several drops or major splash, the category becomes exposure code two.

4. Now if the contact was percutaneous and there was needle injury, the category is exposure code two.

5. If it was from a large-bore hollow needle, the category is exposure code three.

\section{Step 2: Determine HIV status code}

1. If the exposure source is HIV negative, no post exposure prophylaxis is required but if HIV positive, we have to check about low titer or high titer.

2. Low-titer exposures are asymptomatic patients with high CD4 counts: These can be placed in HIV status code one. High-titer exposures are patients with primary HIV infection having low CD4 counts. These are labelled as HIV status code two.

3. If HIV status is unknown or the source is unknown, the HIV status code is also unknown.

Step 3: Match exposure code with HIV status code to determine if any post exposure prophylaxis is indicated

Post exposure prophylaxis recommendations are discussed below.

1. Both Exposure and status code one: Post exposure prophylaxis may not require. Exposure type does not pose a known risk. It is a tricky situation. The exposed health care worker and the treating clinician need to decide if the risk for drug toxicity outweighs the benefit of post exposure prophylaxis.

2. Exposure code one and HIV status code two: Consider the basic regimen. Exposure type poses a negligible risk for HIV transmission. Again the exposed health care worker and the treating clinician need to decide if 
the risk for drug toxicity outweighs the benefit of post exposure prophylaxis.

3. Exposure code two and HIV status code one: The basic regimen is recommended and the use of post exposure prophylaxis is very much appropriate in this case.

4. Both Exposure and status code two: the expanded regimen is recommended. Here exposure type represents an increased HIV transmission risk to the exposed person.

5. Exposure code three and HIV status code one or two: the expanded regimen is recommended. Here exposure type signifies an increased risk of HIV transmission.

6. HIV status code unknown and exposure code is two or three: If exposure code is high and the source is unknown, post exposure prophylaxis with basic regimen is recommended.

\section{Prophylaxis for Hepatitis B}

Hepatitis B prophylaxis measures are as follows:

1. The cases where individuals are previously vaccinated with known and good response to vaccine. No therapy required in all such cases.

2. The cases where individuals previously vaccinated and response to vaccine is not known. In this scenario send fresh blood sample for anti-HBS titer to check previous vaccination response and do administer prophylaxis (one dose of $\mathrm{HBIG}=0.06 \mathrm{~mL}$ per $\mathrm{kg}$ intramuscular); booster dose of vaccination is also required.

3. The cases where individuals are unvaccinated. Immediately inject them with one dose of HBIG and initiate vaccination series for three injections.

There is no known effective post exposure prophylaxis for hepatitis $\mathrm{C}$.

\section{CONCLUSION:}

In our setup occupational exposure to blood and body fluids due to needle stick and sharp related injuries is increasing among health care workers. Phlebotomy procedure and recapping of needles after drawing of blood are commonest procedures, leading to needle stick injuries. A system can be designed for initial training of safety precautions. Awareness campaigns regarding hazards of sharps and needle stick injuries can be introduced in hospital settings. Standard operating procedures intended at prevention from such injuries can be framed. There can be a reporting mechanism by introduction of incident reporting forms of all such injuries. $\mathrm{HBV}$ vaccine has proven to be highly effective in preventing infection in workers exposed to HBV. To date no vaccine exists to prevent $\mathrm{HCV}$ or HIV infection.

\section{REFERENCES:}

1. Hooper, C. (2013). The ethics of blood test after needlestick injury. Retrieved

2. Ethan, C. (2012, Oct) Unconsented HIV Testing in Cases of Occupational Exposure: Ethics, Law, and Policy.

3. Aslam, M. (2010). Needle stick injuries among health care workers of public sector tertiary care hospitals of Karachi. Journal of the College of Physicians and Surgeons Pakistan, 20(3), 150-3.

4. Sultana, A. (2014). Needle Stick/Sharp Injuries in Health Care Workers. Journal of Rawalpindi Medical College. 18(1):133-13.

5. (2004). Centre for Disease Control and Prevention. Workbook for Designing, Implementing and Evaluating a Sharp Injury Prevention Program. Atlanta: US Department of Health and Human Services.

6. Hsieh, W. (2006). Occupational blood and infectious body fluid exposures in a teaching hospital: a three year review. $J$ Microbiol Immunol Infect 39:321-7

7. Aziz, S. (2002). Prevlence of HIV, Hepatitis B and C amongst Health Workers of Civil Hospital Karachi. J Pak Med Assoc. 52:92-95

8. Khurram, M. (2011). Needlestick injuries: A survey of doctors working at tertiary care hospitals of Rawalpindi. JPMA. 61(1) : 63-65

9. (2002) Clinical practice guidelines for needle stick injury. Retrieved from https://www.rch.org.au/clinical-guide/ guideline_index/Needle_stick_injury/

10. Megan, A Stobart-Gallagher.(2017) Needle-stick guideline 